\title{
Research on the Application of Computer Virtual Simulation Technology in 3D Animation
}

\author{
Lian Ding \\ Chengdu Agricultural College \\ 12823666@qq.com
}

Keywords: Computer, Virtual Simulation Technology, 3d Animation, Production, Application

\begin{abstract}
Virtual simulation technology is also called "virtual reality technology" and "simulation technology ", mainly through the virtual system to imitate another real system of an application technology. With the rapid development of information technology today, virtual simulation technology has become a unique technology system, which makes great contribution to the development and progress of mechanical manufacturing, scientific experiments, mathematical reasoning, exploration of natural objective laws and animation design. This paper briefly expounds the virtual simulation technology and its basic teaching requirements, analyzes the internal relationship between computer virtual simulation technology and 3D animation production technology, and probes into the application strategy of computer virtual simulation technology in 3D animation production in depth..
\end{abstract}

\section{Introduction}

Virtual simulation technology is a unique form of technology in modern society, which involves many technical fields, such as human-computer interaction technology, computer graphics theory, sensing technology, artificial intelligence technology and so on. It can make up a three-dimensional artificial environment by means of computer equipment, system software and sensor together, and make people feel immersive through this three-dimensional picture. Virtual simulation technology has certain interaction, lifelike, illusory, immersive, animation designers can grasp this feature, flexible use of virtual simulation technology, comprehensive design, production of threedimensional animation, improve the authenticity of three-dimensional animation scene, Promote the improvement and development of the field of 3D animation design and production in China.

\section{Virtual Simulation Technology and Basic Features}

\subsection{What Is Virtual Simulation Technology}

Virtual simulation technology is a form of technology that imitates another system through one system. It is a computer system that can create and experience the space of virtual world. In the virtual world created by virtual simulation technology, it can reproduce some things in the real world, such as buildings, vehicles, roads and so on, which can also be conceived as the world, such as the "Wonderland" in "Alice's Wandering Wonderland ". People can interact and connect with the virtual world through a variety of perception channels, such as vision, hearing, smell and so on.

Through the data glove, helmet display and other related equipment, it can provide people with a three-dimensional cross section of virtual and reality interaction, through directly participating in exploring the simulation object, exploring the environment in which it is located, to produce a certain sense of immersion, its most representative technical form is "VR technology ", using artificial intelligence technology, simulation technology, microelectronics technology, information technology, multimedia technology, stereo display technology to realize the reproduction of virtual simulation world. 


\subsection{Basic Features of Virtual Simulation Technology}

First, immersion. In the virtual simulation technology system, people can be able to produce the feeling of "immersive" through a variety of perception channels, such as hearing, vision, touch, smell and so on, and immerse themselves in the virtual world and become a part of it.

Second, interactive features. In the virtual simulation system, people can act on people, control the environment through people, and control the environmental changes in the virtual world through body movements, their own language and so on. The environment in the virtual world will give some feedback on human behavior, such as:" people "pick a flower in the virtual world environment, and the flower in the virtual world will be in the hands of" people ", not the flower stem.

Third, illusory features. In virtual simulation systems, the environment in the virtual world is not real, but illusory, it is a world that people use computer technology to simulate with a variety of other technologies; people can simulate the past objective world that has emerged, such as the Qin Dynasty, the Roman period, and so on; also can simulate the world that does not exist and may appear in the future, such as the interstellar world; but also can imitate a variety of non-existent worlds, such as the real world[1].

Fourth, lifelike features. In the virtual simulation system, by constructing the virtual environment, it can promote the popularity to produce the feeling which is very similar to that in the objective real world, and promote people to produce the realistic feeling, just like in the real world. When people act naturally in the virtual world, such as physical action, the environment of the virtual world reacts in accordance with the objective law of the real world, for example, by giving force to an object in the virtual world, the object will move and produce acceleration along the direction of the force.

\section{The Intrinsic Relation Between Computer Virtual Simulation and 3D Animation}

There are some differences between computer virtual reality technology and three-dimensional animation production technology. Three-dimensional animation is mainly through computer program, pre-processing the picture, through the continuous play of a large number of still state of the photo form animation picture, but not interactive and interactive; in the process of threedimensional animation production, people can only receive the picture created by the animation designer in the work, feel the emotion and meaning that the animation designer wants to express, but cannot produce independent emotion and information. However, virtual simulation technology is to help people transmit dynamic information, use computer technology to process and calculate dynamic information, provide people with tactile, auditory and visual perception, and promote people to be stimulated by perception and produce real will. It can be seen from this that the obvious difference between virtual reality technology and 3D animation technology is whether it is interactive or not, and the virtual simulation technology can also respond to people's actual action in a relatively short time to meet people's real needs, which is also not available in 3D animation production technology.[2].

There is a certain connection between computer virtual simulation technology and 3D animation production technology. Virtual simulation technology is a 3D model constructed on the basis of virtual world. The internal heat source of the design will use Maya technology,3D MAX technology and other 3D animation software to construct a virtual world model. In addition, the threedimensional animation production technology and the virtual simulation technology have certain similarity in the use method and the use process, the designer can use the virtual animation production technology, realizes the free interaction between the two, through the virtual simulation technology extends the 3D animation technology, impels the two to form the complementary, thus enhances the 3D animation work presentation effect, cannot only enhance the animation artistic performance ability, but also provides the good, the real interaction experience for the people[3]. 


\section{The Application Strategy of Computer Virtual Simulation Technology in 3D Anmation Production}

\subsection{Build 3d Animation Model to Enhance the Reality of the Model}

Using computer virtual simulation technology in 3D animation production, it can be applied to construct 3D animation model, and improve the reality of virtual world model through virtual simulation technology. Designers should grasp the design and production of the model, focus on the task model creation and animation scene design work, make full use of the virtual simulation technology of human-computer interaction function, improve the flexibility of characters; designers should collect a large number of characters related information, shooting and animation scenerelated natural scenery, forming a data reserve; in the use of virtual simulation technology modeling (figure 1), highly restore the real scene, but also use three-dimensional coordinates to achieve threedimensional data reserve, improve the authenticity of the model.

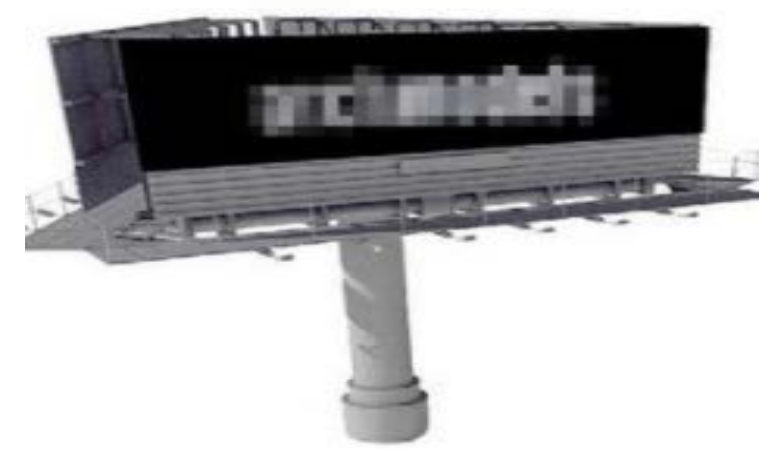

Figure 1 Three-dimensional spinal model

\subsection{Capture the Action of the Animation, Enhance the Scene of the Animation}

Using computer virtual simulation technology in 3D animation production, we can use virtual simulation technology to capture animation action, improve the consistency of 3D animation picture, and promote animation to produce a strong sense of scene. In the process of making 3D animation, the design staff should follow the moving route of the animated object, use the virtual simulation technology to capture dynamically, combine the animation design data after processing by computer, adjust the moving route and state of the object, ensure that the lens can be placed in any place in the animation production, and enlarge and observe one of the moving points to shorten the distance between people and the virtual world, so that people can be immersed in the virtual animation picture (figure 2).

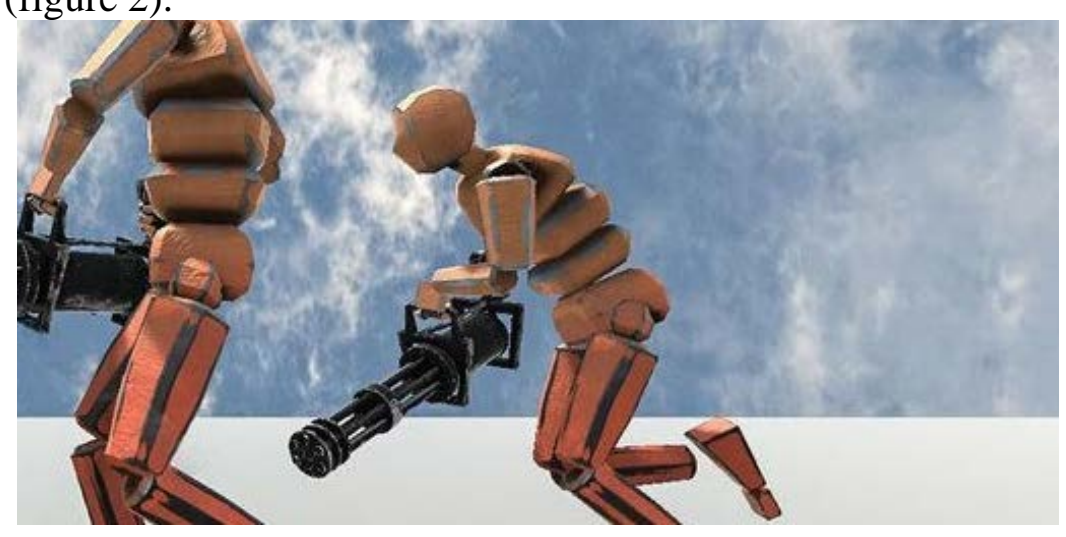

Figure 2 Machine gun human motion capture

\subsection{Mediating the Animation, Optimizing the Processing of 3D Animation Data}

In the production of 3D animation, computer virtual simulation technology can be used to mediate the details of 3D animation, optimize the processing of $3 \mathrm{D}$ animation design and 
production issued, improve the authenticity and infectivity of the picture. The design staff should master all kinds of 3D animation production techniques, make clear the important role of virtual simulation capture system, and improve the authenticity of virtual simulation scene (figure 3) with the help of picture mediation technology; the design staff can modify the picture repeatedly and adjust the details through different methods such as superposition and insertion, so as to realize the purpose of 3D animation production.[4].

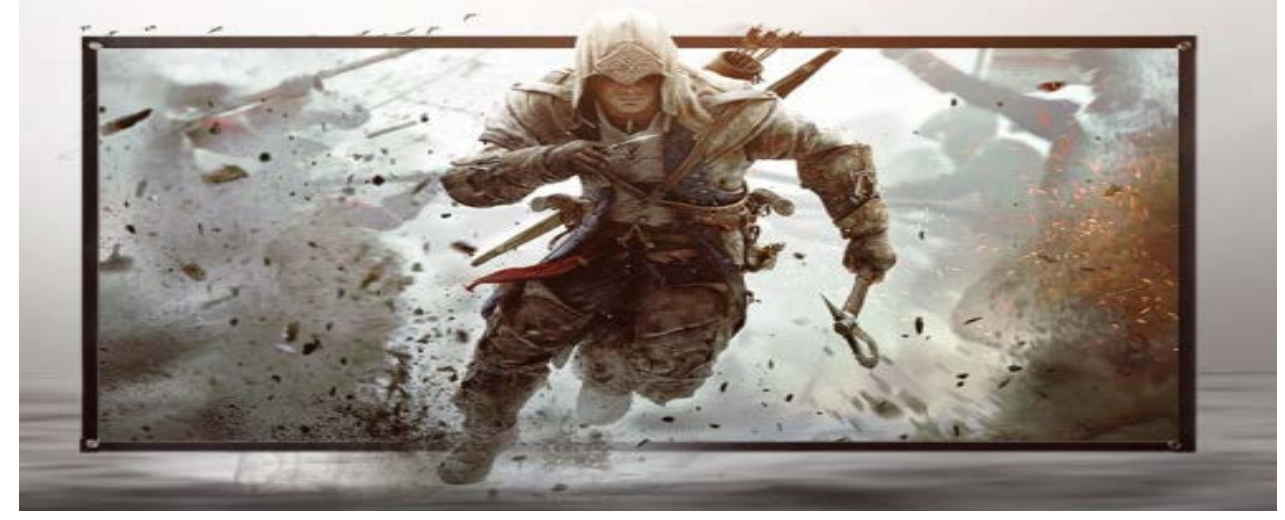

Figure 3 3D Dynamic Picture

\section{Conclusion}

All in all, with the rapid development of science and technology in China, virtual simulation technology has been more and more widely used in various fields, and has initially achieved certain results. In the design and production of 3D animation, the staff should grasp the technical characteristics of virtual simulation technology, explore the essential relationship and difference between virtual simulation technology and 3D animation technology, and grasp the utilization boundary of virtual simulation technology. In the actual design process, the design staff should use the virtual simulation technology to construct the 3D animation model, capture the animation movement, mediate the animation picture, so as to improve the reality of the 3D animation picture, strengthen the scene sense, and improve the dynamic change level of the animation picture.

\section{Acknowledgements}

This research has been financed by The Modern design and culture research center of Sichuan philosophy and Social Sciences Key Research Base in 2018 “Application Research on information visualization design of smart campus in the context of big data -- Taking Chengdu agricultural science and Technology Vocational College as an example” (MD18C003)

\section{References}

[1] Lu Gang. Analysis of the application of virtual reality technology in 3D animation production. Science and Technology Innovation, no. 33, pp. 93-94, 2019.

[2] Yang, Jiangtao. To explore the analysis and application of computer virtual simulation technology. Digital Technology and Applications, vol. 37, no. 10, pp. 54+56, 2019.

[3] Zeng, Longkai. The application of computer virtual simulation technology in 3D animation production. Modern Information Technology, vol. 3, no. 08, pp. 84-85+88, 2019.

[4] Ma, Xinying. Analysis of the application of computer virtual simulation technology in 3D animation production. Technological Innovation Bulletin, vol. 15, no. 03, pp. 161+163, 2018. 